\title{
Symmetry in haptic and in visual shape perception
}

\author{
SOLEDAD BALLESTEROS \\ Universidad Nacional de Educación a Distancia, Madrid, Spain \\ SUSANNA MILLAR \\ University of Oxford, Oxford, England \\ and \\ JOSÉ M. REALES \\ Universidad Nacional de Educación a Distancia, Madrid, Spain
}

\begin{abstract}
Four experiments tested the hypothesis that bilateral symmetry is an incidental encoding property in vision, but can also be elicited as an incidental effect in touch, provided that sufficient spatial reference information is available initially for haptic inputs to be organized spatially. Experiment 1 showed that symmetry facilitated processing in vision, even though the task required judgments of stimulus closure rather than the detection of symmetry. The same task and stimuli failed to show symmetry effects in tactual scanning by one finger (Experiment 2 ). Experiment 3 found facilitating effects for vertically symmetric open stimuli, although not for closed patterns, in two-forefinger exploration when the forefingers had previously been aligned to the body midaxis to provide body-centered spatial reference. The one-finger exploration condition again failed to show symmetry effects. Experiment 4 replicated the facilitating effects of symmetry for open symmetric shapes in tactual exploration by the two (previously aligned) forefingers. Closed shapes again showed no effect. Spatial-reference information, finger movements, and stimulus factors in shape perception by touch are discussed.
\end{abstract}

We know relatively little as yet about the processes that underlie shape perception by touch. The effects of symmetry raise important questions that allow us to explore some of these processes further. Only a few studies have tested for the effects of bilateral symmetry in the tactual perception of two-dimensional (2-D) (raised) shapes. These studies suggest that, without specific training, bilateral symmetry does not have the facilitating effect in tactual processing that has been reported in most studies of bilateral (mirror-image) symmetry in vision.

In vision, bilateral symmetry is often considered the most salient organizational aspect of a stimulus (see, e.g.,

We gratefully acknowledge financial support by the Ministerio de Educacion y Ciencia in conjunction with the British Council. We also wish to thank the Department of Experimental Psychology of the University of Oxford for providing facilities for the work. We are very grateful for the help given to us by the Spanish Blind Organization (ONCE) in producing the stimuli used in the tactual experiments. The research was also supported in part by research Grants DGICYT PB90-0003 and DGICYT PB94-0393 to S.B. and a UNED Graduate Fellowship to J.M.R. It was also supported in part by a research grant to S.M. from the British organization Blindness: Research for Learning, Work and Leisure, which is gratefully acknowledged. We thank D. Ortega for scoring the finger movements. We would also like to record our thanks to the editor and reviewers for their very helpful comments. Portions of the article were presented at 35th annual meeting of the Psychonomic Society, St. Louis, November 1994. Correspondence concerning this article should be addressed to S. Ballesteros, Departamento de Psicología Básica II, Ciudad Universitaria S/N, Universidad Nacional de Educación a Distancia, 28040 Madrid, Spain (e-mail: sballest@cu.uned.es) or to S. Millar, Department of Experimental Psychology, University of Oxford, South Parks Road, Oxford OX1 3UD, England (e-mail: susanna.millar@psy.ox.ac.uk).
Locher \& Nodine, 1973; Mach, 1897; Palmer, 1989; Rock, 1983; Royer, 1981). Bilateral mirror-image symmetry about the vertical axis has usually been reported as being more salient than is symmetry about horizontal or oblique axes (Barlow \& Reeves, 1979; Corballis \& Roldan, 1975; Fisher \& Bornstein, 1982; Munsinger \& Forsman, 1966; Palmer, 1991; Palmer \& Hemenway, 1978; Royer, 1981; Wagemans, Van Gool, \& d'Ydewalle, 1992). Even four-monthold babies have been found to show some appreciation of bilateral symmetry about the vertical axis of shapes (Bornstein, Ferdinandson, \& Gross, 1981; Bornstein \& Krinsky, 1985; Fisher, Ferdinandson, \& Bornstein, 1981).

An early explanation for the advantage of bilateral symmetry in vision assumed that it stems from the bilateral organization of the brain (see, e.g., Corballis \& Roldan, 1975; Julesz, 1971; Mach, 1897). Corballis and Roldan showed that differences in head position did not affect the detection of symmetry and suggested that symmetry detection depends on retinal rather than on gravitational spatial axes. They argued that the advantage that vertical bilateral symmetry had over other orientations was due to a wired-in "template" for vertical symmetry, so that symmetries about other axes required mental rotation. The notion that the advantage of symmetry is due to the structure of the visual system was questioned by Fisher and Bornstein (1982). Unlike Corballis and Roldan, Pashler (1990) found that precuing of the relevant spatial axis produced an advantage for the detection of symmetry. Pashler argued for a frame-of-reference explanation, rather than a mental-rotation explanation, for symmetry effects. It is not clear that Corballis and Roldan's finding necessitates the 
notion of a mental rotation from the gravitational vertical. The discrepant result could have been due to differences in the salience of the potential axis of symmetry in their stimuli.

Locher and Nodine (1973) proposed that subjects use an early organizing code that permits the generation of a feature code on the basis of partial information. The advantage of symmetrical forms is due to the reduction in information from redundant features. Royer (1981) also gave a two-process explanation. Symmetry is encoded integrally with the features of the object, but in detection or decoding tasks, the observer also has to perform a serial hierarchical decomposition of the code. The redundancy of features in bilaterally symmetric shapes makes this process quicker. Two-process theories thus assume early holistic processing. Palmer and Hemenway (1978) included multiple orientation channels as determinants of the process. Specific axes are then evaluated sequentially.

Marr's (1982) computational theory of vision proposed three levels of representation in early visual processing. A "primal sketch" or 2-D image is based on the geometrical distribution and organization of light intensity changes (p. 37). A 2.5-D representation provides a viewer-centered coordinate frame, and the 3-D representation describes shapes in an object-centered coordinate (including volumetric primitives) frame. Marr's use of the terms "representation" and "image" refers primarily to the algorithm that can describe visual computation and its implementation in simulations (Bruce \& Green, 1990). Depth perception can be explained without assuming mediating imagery (see, e.g., Howard \& Rogers, 1995), and many visual processes seem to occur in parallel (Barlow, 1986). Marr's distinctions between viewer-centered, shape-centered, and environmentally based coordinate frames were intended to describe effects on early visual processing. But the distinctions between effects of coordinate reference frames are potentially no less important eventually for an adequate description of haptic processing.

Recent studies on visual symmetry suggest that the frequently reported advantage for symmetry about the vertical axis is confined to bilateral, left-right, mirror-image symmetry (Locher \& Wagemans, 1993; Wagemans et al., 1992). In skewed symmetry, the axis of symmetry in the pattern can lie at any angle to the viewpoint. Vertical-axis symmetry does not have an advantage over the symmetry in other orientations in these conditions (Locher \& Wagemans, 1993; Wagemans, 1995). Symmetry effects can be attenuated by task conditions and cognitive factors (Locher et al., 1993). The findings with complex lines and dots suggest that left-right mirror-image symmetry is detected preattentively, probably by some kind of interaction between the display and spatial organization (Locher \& Wagemans, 1993; Wagemans, 1995). The descriptions propose that visual symmetry is first detected preattentively and then by cognitively controlled attentive processes (Van der Helm \& Leeuwenberg, 1996; Wagemans, 1995).
Visual symmetry effects have thus been explained mainly by two-process theories that assume that symmetry facilitates an initial encoding stage as well as a later stage of deliberate comparison of shape features. The symmetry advantage in the second, or comparison, stage is explained by a reduction of information processing because of the redundancy of symmetric features. Facilitation in the initial stage is variously described as being an integral part of feature encoding, of holistic or preattentive processing, or of initial organizing codes (see above).

Previous evidence suggests that such initial organization is spatial in character. Symmetric/asymmetric organization is a form of (not necessarily conscious) spatial organization. It implies some reference frame, in relation to which features cluster congruently or redundantly. Visual conditions typically provide multiple spatial frames. Such reference information may be viewer-centered, shapecentered, or centered on environmental frames (Marr, 1982). Different frame cues can occur in parallel and redundantly, as well as sequentially. Thus, it is likely that visual shape perception is spatially organized (visuospatial) from the start. If so, symmetric shapes also would have the advantage of being more economically organized early in encoding.

Most visual studies so far have used explicit symmetrydetection tasks. The very nature of explicit tasks precludes there being evidence on initial stages of encoding, because the demand for direct symmetry judgments necessarily draws attention to that dimension. In order to test the suggestion, found in the literature on vision, that symmetric organization is an integral part of early visual-encoding processes, it is necessary to use a perceptual task that does not demand explicit attention to that property. The present study used an indirect perceptual task throughout.

The phrase part of encoding processes is used here in a purely operational sense. It refers solely to the effects that occur as concomitants of processing in tasks that make no explicit demand to detect the property or in tasks that explicitly direct attention to it. No assumptions are made here about the subject's state of awareness. It is assumed that, if an effect occurs as a concomitant of different perceptual tasks, it is part of encoding processes.

In contrast to the case with regard to experiments on symmetry in vision, very few studies have explored symmetry in shape perception by touch (Appelle, 1991). Moreover, the findings of the few studies on touch suggest that bilateral symmetry does not have the same advantage in touch that it has in vision. Walk (1965) found that learning to associate shapes with nonsense syllables was easier for symmetric shapes in vision, but not for those in touch. Locher and Simmons (1978) used eight large, elongated plastic polygons that varied in symmetry and complexity (number of turns) in an explicit symmetry-detection task that followed the visual inspection of similar shapes. Asymmetric shapes were detected more accurately and faster than were symmetric shapes. Simmons and Locher (1979) trained subjects to scan features horizontally across the 
vertical axis of symmetric shapes. The training produced greater sensitivity for symmetric shapes. But it still took less time to decide that a shape was asymmetric, and the advantage of training in symmetry detection did not extend to new shapes. Ballesteros and Reales (1992) found that explicit symmetry judgments of small $(1.8 \times 1.8 \mathrm{~cm})$ raised-line and raised-dot patterns were less accurate and slower than were asymmetry judgments. The difference remained nearly constant for different exposure times. Performance with symmetric shapes was worse $(13 \%)$, even with $2 \mathrm{sec}$ of exploration time (Ballesteros, Manga, Navarredonda, \& Reales, 1993; Ballesteros, Manga, \& Reales, 1997).

Millar (1978) used a tactual matching task that did not demand symmetry detection explicitly. Subjects were better at detecting differences in dot numerosity than they were in discriminating symmetric from asymmetric small raised-dot (braille-like) patterns. This finding was consistent with a series of results that suggested that the discrimination of braille and braille-like patterns depends initially on dot-density differences rather than on global shape coding (Millar, 1978, 1981b, 1994, 1997). Millar explained the findings in terms of the information that the stimulus conditions afford for coding haptic inputs spatially. Novel small raised patterns that derive from a single matrix have few salient (redundant) features that can anchor scanning movements spatially, and the features are too small for their location to be determined by body-centered frames in the absence of environmental cues or of prior experience or training. This explanation suggests that informational conditions, including the size and composition of patterns, influence the amount, type, and redundancy of reference frames that are available for coding haptic inputs as spatially organized shapes (Millar, 1991, 1994, 1977).

In principle, multiple reference frames for spatial coding are available in active touch as well as in vision. A number of geocentric spatial frames can be based on bodycentered (kinesthetic and proprioceptive) information (Paillard, 1991). Spatial frames about the gravitational vertical direction are particularly important (Berthoz, 1991; Howard \& Templeton, 1966; Paillard, 1991). The body midline (z-axis, Howard \& Templeton, 1966) provides a reliable reference axis for head, limb, and hand positions and for hand-movement directions. The relation of hand locations to the body midline affords an initial frame for coding bilateral, redundant features of 3-D symmetric objects spatially in two-handed exploration. An example is the two-handed exploration of unfamiliar 3-D objects that are at fixed locations in line with the subject's body midaxis. It produced an advantage for 3-D symmetric objects (Ballesteros et al., 1997). In the free manipulation of small 3-D stimuli by two hands, the relative spatial locations of shape features (humps and protuberances) can, in principle, be determined instead by the concomitant positions of the thumb and fingers, though this may require extended periods of exploration (Davidson, Abbott, \& Gershenfeld, 1974). For flat objects, the hand itself can act as a spatial reference frame. The distance between felt points can be gauged by the position of the spread-out fingers with respect to each other or to the palm (Katz, 1925, 1989; Krueger, 1982).

For 2-D raised-line and raised-dot stimuli there has long been good evidence that prior information and tactual experience are needed for the systematic scanning that determines adequate shape recognition (Berla \& Butterfield, 1977; Davidson, 1972; Foulke \& Warm, 1967). The question is, why is experience needed and what is the prior information that permits tactual scanning of 2-D patterns to become systematic? The explanation suggested here is that the relevant prior experience or instruction involves some form of spatial-reference information. In the absence of vision, external (environmental) reference cues must depend on distance senses (e.g., sound, smell) that are not often useful in providing spatial cues to locate and organize the features of small 2-D patterns. Thus, shape coding initially depends more on the spatial organization inputs from touch and movement in relation to body-centered spatial frames (Millar, 1981a, 1981b, 1985, 1994). In-line and out-of-line body positions influenced the recall of the extent and direction of hand movements in tracing a straight line (Millar, 1985). Thus, spatial frames are needed to locate and anchor the direction of scanning movements and to organize touch cues spatially as shapes.

Shape symmetry implies some spatial axis or axes in relation to which the features in a pattern are congruent or cluster redundantly. Relevant spatially segregated or organized clusters seem to be available early in visual perception, though cognitive and tasks factors also affect processing (see above). The previous findings on touch suggest that such information is not immediately available for coding novel small 2-D patterns as spatially organized shapes, prior to learning or experience. Shape properties, such as symmetry, are therefore not part of the initial encoding processes. This hypothesis explains the discrepancies in previous findings on symmetry in touch by the amount and kind of available reference information. If spatial information is minimal or lacking, shape properties (including symmetry) have minimal or no effects. Tasks that demand explicit symmetry judgments are solved by a knowledge-based search for the relevant properties, including a search for reference axes in relation to which congruence is tested. In such conditions, asymmetric patterns are easier to detect, because scanning need not be exhaustive. The detection of symmetry, in that case, involves explicit instructions for the search, specific training in spatially organized scanning, or prior spatial-location information. The hypothesis, here called the reference hypothesis for convenience, agrees substantially with the proposal by Locher and Simmons (1978) that tactual scanning interacts with the stimulus and task factors. It differs with their proposal in that it places more explicit emphasis on the contribution of available spatial-reference information in the spatial organization of inputs from touch and movement.

The effects of symmetry in touch have theoretical implications for models of tactual perception. A better un- 
derstanding of these processes is needed, particularly in the case of small 2-D raised-line patterns, because patterns of that kind are often used as symbols in tactual maps. If symmetry facilitates visual processing even when the task does not demand direct judgments of symmetry, it is assumed to be part of encoding processes. It is assumed here that any advantage of symmetric over asymmetric small 2-D shapes in touch depends on providing adequate spatial-reference cues.

It is thus suggested that the perception of shape symmetry in touch depends on the amount and type of spatialreference information that is initially available to the subject from all sources (Millar, 1994, 1997). This hypothesis implies that, when there is adequate initial spatial-reference information for shape coding to take place, symmetric organization affects touch also and facilitates processing. The reference hypothesis, therefore, predicts that conditions that provide sufficiently fine-tuned spatial-reference information for shape coding by touch should also produce an advantage for symmetric patterns, without there being explicit instructions to detect symmetry.

The hypothesis thus makes three main predictions. In vision, an indirect perceptual task will produce the usual advantage of bilaterally symmetric 2-D shapes, because symmetric or asymmetric clustering of shape features is an integral part of early visuospatial information and organization. In touch, by contrast, there is a paucity of spatial-reference information when exploring a small, unfamiliar 2-D pattern by one finger, without the use of vision. There is, thus, little initial information for coding the patterns spatially as global shapes. Since symmetric organization is spatial in character, symmetry will not affect processing in these conditions, unless explicit symmetry judgments are required. The prediction with respect to touch is, therefore, that an indirect perceptual task will produce symmetry effects only when specific spatial-reference information is provided for initial encoding. The hypothesis thus also predicts that, when spatial-reference information is made more specific, symmetry will have facilitating effects on touch with an indirect task.

In the present study, four experiments were undertaken to test the hypotheses that symmetry is an early encoding property for 2-D shapes in vision, that it is not an early encoding property in touch, and that providing body-centered bilateral reference cues produces an advantage also for bilaterally symmetric stimuli in touch. The first experiment tests the hypothesis with respect to vision in order to compare symmetry effects in an indirect perceptual task with previous findings. The subsequent three experiments test the hypotheses with respect to touch. The stimuli and task conditions were designed principally for ease of use with touch.

Explicit symmetry-judgment tasks, which have mainly been used in previous studies, cannot provide evidence concerning processes early in encoding. To test whether symmetry acts as an early encoding property in either vision or touch thus required an indirect perceptual task. Detecting a closed shape within patterns was used as the in- direct task, because it is easy for touch as well as for vision. Visual studies on bilateral symmetry have generally shown an advantage for symmetry about the vertical axis (i.e., left-right mirror images). Orientation was, therefore, included as a factor in all four experiments. To our knowledge, no other study on symmetry in touch has varied stimulus orientation systematically.

To test the hypotheses with respect to touch, previous task conditions, which provide few spatial-reference cues, were compared with conditions that specifically provide spatial-reference information. Previous informational conditions for touch were replicated in Experiment 2 by using scanning, with unlimited exploration time, by one forefinger that had not been specifically centered. Spatialreference information was provided in Experiments 3 and 4 by aligning the two forefingers with the body midaxis of the subject in order to make body-centered frame cues more salient. The use of two fingers of the same hand to scan the outline shapes of familiar objects does not improve recognition in the way that enlarging the visual field by an analogous amount does (Loomis, Klatzky, \& Lederman, 1991). However, aligning the forefingers of the two hands in parallel and positioning them in direct alignment with the midpoint of the body midaxis should afford more finely tuned body-centered reference information. Such reference should, therefore, facilitate the spatial coding of bilateral (redundant) features of symmetric shapes.

Consequently, in Experiments 3 and 4, the two forefingers of the subject were carefully positioned directly above the stimulus shape, in alignment with the midaxis of the body. The two forefingers moved down perpendicularly to touch the shape, which started the trial. Their location was thus in direct vertical (tabletop projected) alignment with the midpoint of the subject's body. Our intention was to provide body-centered reference cues for the locations of the two forefingers that were to be used in scanning. It was expected that the frame cues would produce a symmetry advantage also in touch. In the third experiment, the two-forefinger condition was compared with the one-finger condition. In order to provide some redundancy of reference cues for touch, we designed the stimuli in the first three experiments in a way that incorporated shape-centered orientation cues. A further test with the two-forefinger tactual condition, using one stimulus set in three orientations without specific shapecentered cues, was conducted in the fourth experiment.

\section{EXPERIMENT 1}

The hypothesis under test was that symmetry facilitates the processing of visual patterns, even when the task does not direct attention to that property. The indirect perceptual task was the detection of a closed shape within a pattern. Since vertical-axis symmetry was found to be most salient in previous reports on bilateral symmetry in vision, it was expected that this would obtain also with the indirect perceptual task used here. 


\section{Method}

Subjects. The subjects were 36 unpaid volunteers receiving course credit at Madrid University (UNED) who were naive as to the purpose of the experiment.

Stimulus sets. The visual stimuli were line drawings, from which the raised-line tactual stimuli were produced for the experiments on touch. The shapes were carefully designed so as to be suitable for discrimination by touch as raised lines on thermoform. The criteria for the design of the symmetric and asymmetric stimuli were thus based on a number of practical considerations with respect to their use by touch, including the suitability of stimuli of this kind as symbols in tactual maps.

The consideration of size and clarity for touch was paramount. All our stimuli were generated from a $3 \times 3(2 \times 2 \mathrm{~cm})$ dot matrix, somewhat larger than the $3 \times 3$ matrix used by Foulke and Warm (1967), which showed very accurate tactual responses. The patterns were generated by connecting lines between the dots within the matrix. The resulting visual line drawings of the patterns were professionally produced by the Spanish Organization for the Blind (ONCE) as raised-line patterns on thermoform for the experiments on touch.

Detection of a closed figure within line patterns is probably the easiest perceptual judgment, and we found it to be so for touch (see below). We therefore selected stimuli that were small enough not to require large arm movements but were sufficiently clear for easy tactual discrimination. Because the indirect task demanded that half of all the patterns should contain a closed shape, the dot matrix was spaced more widely than were the Foulke and Warm (1967) patterns. The total area $(2 \times 2 \mathrm{~cm})$ was large enough to generate patterns that contained easily felt closed shapes. The minimum length of the lines $(10 \mathrm{~mm})$ and the surface area of the closed shape $(10 \times 10 \mathrm{~mm})$ that was contained within patterns was thus larger than the $(6.2 \mathrm{~mm})$ braille cell.

Half of the stimuli were symmetric, and the other half were asymmetric; half of the symmetric and half of the asymmetric stimuli contained a closed shape. For convenience, the stimuli that contained a closed shape will be called closed stimuli. The other half of symmetric and asymmetric stimuli did not contain a closed shape. These stimuli will be referred to as open stimuli.

Orientation was included as a factor in order to test for the same advantage of vertical-axis bilateral symmetry for touch that had often been reported for vision. The stimuli were therefore designed to be oriented in three orientations-vertical, horizontal, and oblique-relative to the rectangular coordinates of the presentation card. As in most previous studies of symmetry, stimulus patterns did not necessarily include the axis of symmetry as an actual midline feature of the stimuli.

We assumed that, for touch, the features of vertical stimuli could be specified most easily by reference to the body-centered frame. However, we also considered that the orientation of a small $(2 \times$ $2 \mathrm{~cm}$ ) shape, relative to body-centered frames, is likely to be less salient than it is in vision. Vision provides external environmentalbut also shape-centered-as well as body-centered frame cues. There is much less redundancy of frame cues in touch than in vision. Our hunch was that, in touch, the orientation of the shapes only in terms of body-centered frames would not be sufficient for shapes to be oriented accurately. It was, therefore, considered important to ensure that information about the relevant orientation of a shape was also available redundantly as a shape-centered cue in touch. Each stimulus was therefore designed so that the direction of the main line or lines in the patterns ran in the relevant orientation.

The stimuli for testing orientation effects in the first three experiments were thus designed specifically to have as many lines as possible in the relevant orientations within the stimuli, in order to provide redundant, shape-centered orientational information relative to body-centered frames. This further restricted the choice of possible designs from the $3 \times 3$ dot matrix that was the basis for all open and closed symmetric and asymmetric stimuli.

It had been intended also to provide an additional shape-centered spatial cue for the relevant orientations of the tactual stimuli by using an arrow-like directional marking, on one of the main lines of orientation, as a potential directional axis in half of all of the stimuli. Schiff, Kaufer, and Mosak (1966) produced such directional arrows in raised lines and showed that subjects interpreted the directional nature of the resulting lines very accurately. Unfortunately, the equipment available to ONCE to produce multiple, accurate raised patterns on thermoform (geared to braille and tactual maps) was capable of producing only lines of dots and smooth lines, and not lines of arrows. It was nevertheless decided to include the stimuli with dotted lines, as originally intended, because dotted lines have sometimes been found useful for touch (see, e.g., Loomis, 1981; but see Lederman \& Kinch, 1979). To test for the effects of the additional shape-centered orientation cue-stimuli with smooth raised lines, as opposed to stimuli with raised-dot lines - therefore constituted a further factor in the experiments. Half of all of the symmetric and asymmetric open and closed shapes in the three (vertical, horizontal, and oblique) orientations, therefore, contained dotted-lines in relevant orientations; the other half of the stimuli in all conditions only contained smooth lines. In fact, the variable - from now on called the smooth-line/dot-line (texture) factor-produced no consistent effects. The results for the further smooth-line/dot-line factor are, nevertheless, reported in the relevant studies for the sake of completeness.

An important restriction in generating all stimuli was that each stimulus in all conditions should occupy about the same surface area. The stimuli in all conditions were also to be comparable in complexity, which, in the context of the present stimuli, refers to the number of connecting lines that composed each pattern. The restriction meant that each pattern should contain no more and no fewer than five or six connecting lines. Equating the number of connecting lines generated from one $3 \times 3$ matrix was here equivalent to equating the physical complexity of stimuli in different conditions. The number of lines composing the shapes did not differ between any of the factorial conditions to be tested.

There was thus a total number of 144 stimuli, 6 stimuli in each (symmetric/asymmetric $\times$ open/closed $\times$ vertical/horizontal/ oblique orientation $\times$ smooth-line/dot-line) condition. The number of stimuli and trials were equal (6) in each of the experimental conditions. The 144 stimuli were presented on a computer monitor. The order of stimuli was randomized over all factors and conditions, so that each subject received a new order of stimuli. Examples of stimuli in every condition are shown in Figure 1.

Equipment. The patterns were exposed singly on an IBM System $/ 2$ computer screen. The screen contrast was good. Stimuli were presented black on white (Michelson contrast, $C=\left[\left(L_{\max }-\right.\right.$ $\left.\left.L_{\min }\right) /\left(L_{\max }+L_{\min }\right)\right]$ of $\left.0.7\left(L_{\max }=159 \mathrm{~cd} / \mathrm{m}^{2}, L_{\min }=28 \mathrm{~cd} / \mathrm{m}^{2}\right)\right)$. The system was interfaced with a voice key (Lafayette 63040) for the appropriate voiced response. The experiment was programmed in Pascal.

Design and Procedure. A subjects $\times$ symmetry (symmetric/ asymmetric) $\times$ orientation $(\mathrm{V} / \mathrm{H} / \mathrm{O}) \times$ open/closed stimuli $\times$ smoothline/dot-line repeated-measures design was used. The task was to judge whether the presented pattern was open (open stimuli) or contained a closed shape (closed stimuli) by saying "abierto" (open) or "cerrado" (closed).

The subjects were tested singly in the laboratory. They were seated directly in front of the screen, at a distance of about $50 \mathrm{~cm}$. The visual angle subtended by the shape was $2.29^{\circ}$. The interstimulus interval was self-paced. All stimuli appeared in the center of the screen. To start a trial, the subject pressed a key which produced a cross in the center of the monitor. The subjects were instructed to fixate this cue. The fixation cue was replaced after $500 \mathrm{msec}$ by a 
UNTEXTURED LINE

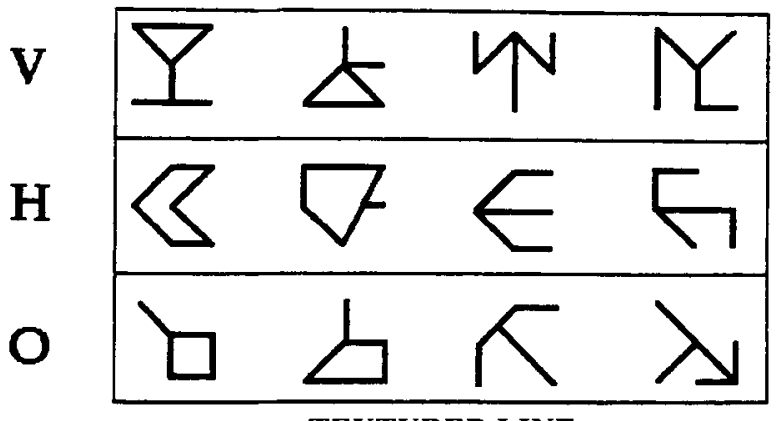

TEXTURED LINE

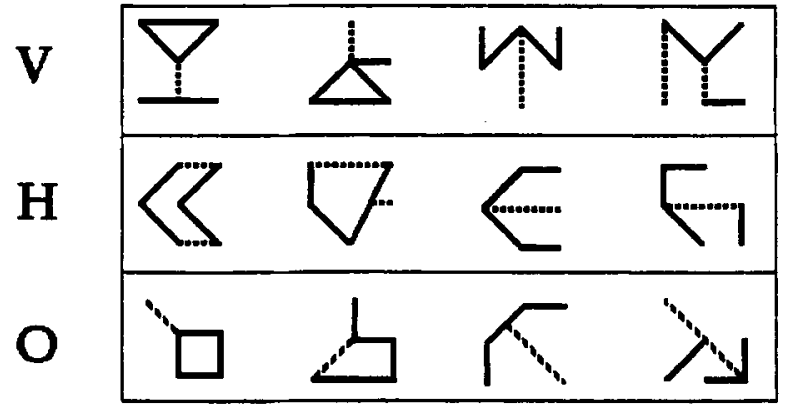

Figure 1. Examples of sets of stimuli in Experiments 1, 2, and 3. The top sets show smooth-line (untextured) stimuli. The bottom sets show the identical stimuli with dotted (textured) lines in the relevant $V$ (vertical), $H$ (horizontal), and $O$ (oblique) orientations. The sequence of shapes in each $V, H$, and $O$ orientation is shown as follows: (1) closed symmetric, (2) closed asymmetric, (3) open symmetric, and (4) open asymmetric shapes. (The full set of 144 stimuli is available on request from the first two authors.)

stimulus shape, which was exposed for $116 \mathrm{msec}$, after which a line mask appeared at the same position. Exposure time was restricted to $116 \mathrm{msec}$, after a pilot study had shown that this would produce an accuracy level between $70 \%$ and $80 \%$, to avoid ceiling and floor effects. The mask remained on the screen until the subject produced an oral response. The voice key was attached to the subject's collar. The subject's oral response stopped the timer so that response times (RTs) were recorded automatically. The trigger time for the voice key was not distinguishable between the two response words.

The subjects received 6 practice trials, starting with exposure times above $116 \mathrm{msec}$. The exposure time was gradually reduced to $116 \mathrm{msec}$ during these trials. The testing session lasted for about $30 \mathrm{~min}$. The subjects had a rest for about $3 \mathrm{sec}$ after 72 trials.

\section{Results}

Errors. The mean percentages of errors (overall, $18.12 \%$ ) are shown in Table 1A. The relevant analysis of variance (ANOVA) showed a significant effect of symmetry $\left[F(1,35)=5.329, M S_{\mathrm{e}}=0.71, p<.03\right]$. Judgments of symmetric stimuli were more accurate than those of asymmetric stimuli (17\% vs. $19 \%)$, which is consistent with the hypothesis that symmetry facilitates visual recognition. Orientation was also significant $[F(2,70)=4.184$, $\left.M S_{\mathrm{e}}=1.29, p<.02\right]$. As predicted for vision, vertical orientation was the most accurate overall $(15.66 \%)$ and differed significantly from horizontals $(19.16 \%, p<.05)$.
Judgments of oblique stimuli were also less accurate than were those of vertical stimuli overall, but that difference did not reach statistical significance ( $15.66 \%$ vs. $20.16 \%$ ).

The interaction between symmetry and orientation was significant $\left[F(2,70)=6.233, M S_{\mathrm{e}}=0.64, p<.004\right]$. Judgments of stimuli with symmetry about the vertical axis were significantly more accurate than were those of stimuli with vertical asymmetry $(p<.05)$. This was also the case for the obliques $(p<.05)$, which seemed to behave similarly to verticals. For horizontals, the difference was in the opposite direction but was not significant. A similar finding has been reported for vision previously (Corballis \& Roldan, 1975).

Judgments of closed stimuli were significantly more accurate than were those of open stimuli $[F(1,35)=22.65$, $\left.M S_{\mathrm{e}}=5.46, p<.0001\right]$. The only other significant effect was a higher order interaction of symmetry/asymmetry with open/closed stimuli and smooth-lines/dot-lines $\left[F(1,35)=12.90, M S_{\mathrm{e}}=0.403, p<.001\right]$. Smooth lines produced fewer errors for symmetric open shapes than did dot lines $(p<.05)$. But that was not the case for closed shapes.

Response times. Mean RTs for correct trials are shown in Table 1B. The relevant ANOVA showed that closed stimuli took significantly less time ( $77 \mathrm{msec})$ than did open stimuli $\left[F(1,35)=16.625, M S_{\mathrm{e}}=0.077, p<.0003\right]$. Symmetric stimuli were faster $(22 \mathrm{msec})$ than asymmetric stimuli but not significantly so $(p=.12)$. Our exposure times were made extremely short $(116 \mathrm{msec})$. The effect of symmetry thus showed significantly only in the accuracy measure, although the same trend was evident in RTs also.

Supplementary (control) test. As a further control for testing symmetry effects in subsequent tactual experiments, a supplementary visual test used the actual raisedline tactual (thermoform) stimuli, presented on the flat tactual testing board on the table at which the subject was sitting, precisely as for the later tactual experiments. Subjects looked at the stimuli through a liquid crystal window in the presenting apparatus. No visual fixation point was used in this experiment. In fact, the raised-line thermoform tactual stimuli provided too little contrast to be seen, except by illuminating them from the side in such a way that the raised lines produced visible shadows of the shapes. The viewing conditions were thus poor and were more like the skewed conditions that produce orientation effects (see, e.g., Wagemans, 1995) that are unlike the bilaterally symmetric conditions that we were trying to reproduce for touch. The symmetry effects were, nevertheless, of interest, because stimuli in the subsequent experiments on touch used the flat (tabletop) presentation. The control test was thus run solely to ensure that the visual symmetry effects in Experiment 1 were not simply due to the upright stimulus presentation.

The presenting apparatus provided tachistoscopic (computer-controlled) visual exposures of $40 \mathrm{msec}$. Thirty-six subjects were tested, as before. They received 10 practice trials, starting with a $200-$ msec exposure time, 
Table 1A

Mean Percentage of Errors for Types of Stimuli (Open/Closed), Symmetric Versus Asymmetric Conditions in Vertical, Horizontal, and Oblique Orientations With Textured (T) and Untextured (NT) Line

\begin{tabular}{|c|c|c|c|c|c|c|c|c|c|}
\hline \multirow[b]{3}{*}{ Orientation } & \multicolumn{4}{|c|}{ Open } & \multicolumn{4}{|c|}{ Closed } & \multirow[b]{3}{*}{$M$} \\
\hline & \multicolumn{2}{|c|}{ Symmetric } & \multicolumn{2}{|c|}{ Asymmetric } & \multicolumn{2}{|c|}{ Symmetric } & \multicolumn{2}{|c|}{ Asymmetric } & \\
\hline & $\mathrm{T}$ & NT & $T$ & NT & $\mathrm{T}$ & NT & $\mathrm{T}$ & NT & \\
\hline Vertical & 18.50 & 14.83 & 24.99 & 26.32 & 8.83 & 16.66 & 9.66 & 10.66 & 15.66 \\
\hline Horizontal & 31.98 & 23.16 & 22.15 & 28.65 & 17.16 & 13.83 & 17.16 & 8.33 & 19.16 \\
\hline Oblique & 25.49 & 23.16 & 29.49 & 26.32 & 8.82 & 7.83 & 15.66 & 12.50 & 20.16 \\
\hline$M$ & 25.15 & 20.16 & 25.15 & 27.65 & 11.49 & 11.16 & 14.16 & 10.50 & 18.16 \\
\hline
\end{tabular}

Table $1 B$

Mean Response Times (in Milliseconds) for Types of Stimuli (Open/Closed), Symmetric Versus Asymmetric Conditions in Vertical, Horizontal, and Oblique Orientations With Textured (T) and Untextured (N') Line

\begin{tabular}{|c|c|c|c|c|c|c|c|c|c|}
\hline \multirow[b]{3}{*}{ Orientation } & \multicolumn{4}{|c|}{ Open } & \multicolumn{4}{|c|}{ Closed } & \multirow[b]{3}{*}{$M$} \\
\hline & \multicolumn{2}{|c|}{ Symmetric } & \multicolumn{2}{|c|}{ Asymmetric } & \multicolumn{2}{|c|}{ Symmetric } & \multicolumn{2}{|c|}{ Asymmetric } & \\
\hline & $\mathrm{T}$ & NT & $\mathrm{T}$ & NT & $\mathrm{T}$ & NT & $\mathbf{T}$ & NT & \\
\hline Vertical & 1,028 & 1,001 & 926 & 1,009 & 897 & 935 & 894 & 892 & 948 \\
\hline Horizontal & 955 & 953 & 941 & 1,021 & 927 & 899 & 914 & 889 & 937 \\
\hline Oblique & 957 & 978 & 989 & 967 & 1,016 & 849 & 851 & 841 & 931 \\
\hline$M$ & 980 & 978 & 952 & 999 & 947 & 894 & 887 & 874 & 938 \\
\hline
\end{tabular}

which was progressively reduced to $40 \mathrm{msec}$. In all other respects, the task, design, and procedures were the same as before.

Symmetry had a highly significant main effect on accuracy $\left[F(1,35)=36.16, M S_{\mathrm{e}}=0.7202, p<.0001\right]$. As before, the indirect perceptual task produced fewer errors for symmetric $(17.17 \%)$ stimuli than for asymmetric $(22.95 \%)$ stimuli.

\section{Discussion}

Bilateral symmetry facilitated visual shape processing, even though the task did not direct attention to that dimension explicitly. The results show an incidental detection of bilateral symmetry in vision, which is consistent with the assumption that bilateral symmetry is part of early encoding processes in visual shape perception.

The interaction that shows a significant advantage of vertical-axis symmetry is similar to previous findings for bilaterally symmetric visual stimuli in tasks that required explicit symmetry judgments (see, e.g., Barlow \& Reeves, 1979; Palmer, 1991; Palmer \& Hemenway, 1978; Royer, 1981). Some studies that used unrestricted viewing times or long $(2,000 \mathrm{msec})$ exposures without masks (Corballis \& Roldan, 1975; Palmer \& Hemenway, 1978; Royer, $1981)$ report the symmetry effect for latency rather than for accuracy measures. It was also clear that the significant main effect of symmetry cannot be attributed to there being an upright, rather than a flat, stimulus presentation.

No explanation is offered here for the higher order interaction that showed that open symmetric stimuli with dot lines produced more errors than did open symmetric stimuli with smooth lines. The dot lines in stimuli had been intended to enhance the stimulus orientation for touch.
The finding does not speak to that. It is possible that texture differences have adverse effects on global processing.

\section{EXPERIMENT 2}

This experiment was run in order to test whether similar bilateral symmetry effects occur for touch in an indirect judgment task with unlimited exploration time. The shapes were the same as those for Experiment 1, but were produced as raised lines on thermoform. The hypothesis was that symmetry effects in touch depend on spatial-reference information early in encoding. It predicts that, if the relation between the exploring finger and the body midaxis is not sufficiently precise initially to serve as a spatial reference frame for coding small 2-D raised-line stimuli as global shapes, stimulus symmetry will not affect tactual processing, even with unlimited exploration time, in cases where the perceptual task does not demand the detection of symmetry explicitly.

\section{Method}

Subjects. Twenty-four new right-handed unpaid volunteers, receiving course credit at the UNED University, participated in this experiment. To avoid over burdening the subjects, 8 subjects were allocated randomly to each of the three $(\mathrm{V} / \mathrm{H} / \mathrm{O})$ stimulus orientation conditions that were to be tested. The subjects were tested blindfolded and did not see the stimuli or the apparatus at any time, as was also the case in all subsequent tactual experiments.

Stimuli. The tactual stimuli were generated from the 144 stimulus designs that were described in Experiment 1 (Figure 1). The line drawings were used as the templates to produce the stimuli as raised $(0.7 \mathrm{~mm}$ in height, $2.2 \mathrm{~mm}$ in width, and a minimum of $10 \mathrm{~mm}$ in length) line $(2 \times 2 \mathrm{~cm})$ patterns on (heated) thermoform cards.

The stimulus patterns were centered in the middle of $(14 \times$ $20 \mathrm{~cm}$ ) presentation cards, and these were placed on the flat presen- 
tation board in the recording apparatus in the manner described below. The $(\mathrm{V} / \mathrm{H} / \mathrm{O})$ orientations of the stimuli thus refer to the horizontal tabletop (mid-transverse plane) throughout.

A pilot study was conducted in order to ascertain the probable level of accuracy in detecting closed and open stimuli. Five blindfolded subjects were tested singly. They did not see the apparatus or the stimuli at any time. The results showed that the judgments were easy and accurate.

Recording apparatus. The apparatus consisted of a flat piezoelectric board, interfaced with an IBM System/2 computer that recorded the main data. The board was placed on the table at which the subject was seated. The stimulus cards were placed on the board and were secured by clamps at each side of the board. This ensured that the cards could not be displaced during tactual exploration. The piezosensor was located underneath the board, directly below the middle of the stimulus card. Two inclined mirrors, attached laterally to the right and left sides of the board, allowed the experimenter to observe the subject's (lateral) exploratory movements over the shapes. The finger movements were recorded additionally from above by a video camera that was fixed to the wall facing the subject.

The relevant stimulus card was selected automatically by a computer program that randomized all variables for each trial for every subject. The same program recorded the data for the relevant variables.

Design and Procedures. A symmetry (symmetric/asymmetric) $\times$ orientation $(\mathrm{V} / \mathrm{H} / \mathrm{O}) \times$ open/closed stimuli $\times$ smooth-line/dotline design was used with repeated measures on all factors except orientation. The task, as in Experiment 1, was to judge whether the stimuli were open or closed.

Subjects were right-handed and were asked to use their preferred forefinger to explore the shapes, as was done in the study by Foulke and Warm (1967). A previous study (Ballesteros et al., 1997, Experiment 2) had shown no difference in accuracy and sensitivity $\left(d^{\prime}\right)$ between the left and right forefingers in haptic exploration of very similar stimuli. Since no difference between the hands had been found in similar conditions, scanning with the preferred forefinger was used for one-handed scanning.

The subjects were tested singly in the laboratory. They were seated at the table on which the testing board and stimuli were placed. The stimulus board was on the table directly in front of the seated subject. The experimenter positioned the subject's forefinger above the shape (at a height of approximately $20 \mathrm{~cm}$ ) before the start of the signal. The subjects were informed of the fact that the stimulus was on the table directly below their finger. Neither the finger position nor the movements were constrained.

The subjects were told that they would be shown a series of raisedline drawings. They were to explore each shape with their preferred forefinger and to judge whether the shapes were open or closed. The terms were explained, and the subjects were given a series of six practice trials in order to ensure that they understood the task. They were asked to work as quickly and accurately as possible.

The subject was told to start exploring the stimulus immediately on hearing the starting signal (a tone from the computer). Timing started automatically when the subject touched the stimulus card. Latencies were recorded from first touch to voiced response. Correct and incorrect responses were recorded by the experimenter by pressing the appropriate key on the computer keyboard.

\section{Results}

Errors. There were very few errors overall (5.9\%). The mean percentages of errors in all conditions are shown in Table $2 \mathrm{~A}$. The ANOVA on errors produced only a significant main effect of open/closed stimuli $[F(1,21)=15.76$, $\left.M S_{\mathrm{e}}=0.0197, p<.0007\right]$. The open shapes produced significantly fewer errors $(2.6 \%)$ than did the closed shapes $(9.2 \%)$. No significant effect was found for symmetry/ asymmetry $[F(1,21)=0.665, p=.4]$ or for orientation $(\mathrm{V} / \mathrm{O} / \mathrm{H})[F(2,21)=3.155, p=.063]$, although the latter showed a positive tendency in the expected (better vertical) direction. No other effect or interaction was significant either.

Response latencies. Mean RTs for correct trials are shown in Table 2B. The ANOVA on RTs (in seconds) for correct responses showed no significant effect of either symmetry or orientation. The open/closed stimuli had a significant main effect $\left[F(1,21)=7.30, M S_{\mathrm{e}}=4.004, p<\right.$ $.02]$. Closed shapes (overall mean $=6.44 \mathrm{sec}$ ) took sig-

Table 2A

Mean Percentage of Errors for Types of Stimuli (Open/Closed), Symmetric Versus Asymmetric Conditions in Vertical, Horizontal, and Oblique Orientations With Textured (T) and Untextured (NT) Line

\begin{tabular}{|c|c|c|c|c|c|c|c|c|c|}
\hline \multirow[b]{3}{*}{ Orientation } & \multicolumn{4}{|c|}{ Open } & \multicolumn{4}{|c|}{ Closed } & \multirow[b]{3}{*}{$M$} \\
\hline & \multicolumn{2}{|c|}{ Symmetric } & \multicolumn{2}{|c|}{ Asymmetric } & \multicolumn{2}{|c|}{ Symmetric } & \multicolumn{2}{|c|}{ Asymmetric } & \\
\hline & $\mathrm{T}$ & NT & $\mathrm{T}$ & NT & $\mathrm{T}$ & NT & $\mathrm{T}$ & NT & \\
\hline Vertical & 0.0 & 2.1 & 2.1 & 0.0 & 4.3 & 8.5 & 4.3 & 4.3 & 3.2 \\
\hline Horizontal & 4.3 & 0.0 & 0.0 & 0.0 & 2.1 & 6.4 & 6.4 & 8.4 & 3.4 \\
\hline Oblique & 12.6 & 2.1 & 6.4 & 2.1 & 21.1 & 12.7 & 12.6 & 19.0 & 11.1 \\
\hline$M$ & 5.6 & 1.4 & 2.8 & 0.7 & 9.2 & 9.2 & 7.8 & 10.5 & 5.9 \\
\hline
\end{tabular}

Table 2B

Mean Response Times (in Seconds) for Types of Stimuli (Open/Closed), Symmetric Versus Asymmetric Conditions in Vertical, Horizontal, and Oblique Orientations With Textured (T) and Untextured (NT) Line

\begin{tabular}{|c|c|c|c|c|c|c|c|c|c|}
\hline \multirow[b]{3}{*}{ Orientation } & \multicolumn{4}{|c|}{ Open } & \multicolumn{4}{|c|}{ Closed } & \multirow[b]{3}{*}{$M$} \\
\hline & \multicolumn{2}{|c|}{ Symmetric } & \multicolumn{2}{|c|}{ Asymmetric } & \multicolumn{2}{|c|}{ Symmetric } & \multicolumn{2}{|c|}{ Asymmetric } & \\
\hline & $\mathrm{T}$ & NT & $\mathrm{T}$ & NT & $\mathrm{T}$ & $\mathrm{NT}$ & $T$ & $\mathrm{NT}$ & \\
\hline Vertical & 7.56 & 7.64 & 7.63 & 7.26 & 7.17 & 7.11 & 6.42 & 6.36 & 7.14 \\
\hline Horizontal & 6.34 & 6.82 & 6.03 & 7.30 & 5.81 & 6.47 & 5.35 & 6.50 & 6.33 \\
\hline Oblique & 7.80 & 7.55 & 7.60 & 7.11 & 5.93 & 6.17 & 6.17 & 7.40 & 7.02 \\
\hline$M$ & 7.23 & 7.34 & 7.09 & 7.22 & 6.30 & 6.73 & 5.98 & 6.75 & 6.83 \\
\hline
\end{tabular}


nificantly less time to explore than did open shapes (overall mean $=7.22 \mathrm{sec}$ ).

There were no significant main effects of symmetry/ asymmetry or orientation, and no other factor or interaction was significant.

Analysis of finger movements. An analysis of actual scanning movements was carried out for 12 subjects, 4 in each orientation, randomly selected from the subjects who took part in this experiment. The finger movements recorded on videotape (see above) were scored (blind) by an independent observer in slow-motion and frame-by-frame analysis for each stimulus for each of the 12 subjects.

The orientation of each finger movement was defined relative to the Euclidean coordinates of the surrounding frames of the stimulus card and matrix of the stimulus shape. A vertical (up-down or down-up) finger movement was a straight movement that ran parallel to the vertical axis. To be scored as horizontal (right-left, leftright), the movement paralleled the horizontal axis. Oblique movements (both directions) were straight-line diagonal movements (top-left to bottom-right, top-right to bottomleft, or vice versa). A straight movement was delimited by a visible change in the direction of that movement. Circular movements were scored if the movement proceeded in smooth curved sweeps, usually completing a full circle, rather than by changes in the angle of a straight movement. The directions and changes in direction of exploratory movements in scanning a stimulus were easily visible and quite obvious from the video in frame-by-frame and slowmotion analyses.

The criteria for scoring movements in any one design were to allot one point each for any vertical, horizontal, or oblique movement in either direction and one point for each circular movement. Individual subjects varied considerably in the total number of movements they used for each design. For comparability, scores in each movement

Table 3

Mean Percentage of Coordinated Vertical, Horizontal, Oblique, and Circular Movements for Open and Closed, Symmetric and Asymmetric Types of Stimuli in Vertical, Horizontal, and Oblique Orientations (Experiment 2)

\begin{tabular}{|c|c|c|c|c|}
\hline \multirow[b]{2}{*}{ Movement } & \multicolumn{2}{|c|}{ Open } & \multicolumn{2}{|c|}{ Closed } \\
\hline & $\overline{\text { Symmetric }}$ & Asymmetric & Symmetric & Asymmetric \\
\hline \multicolumn{5}{|c|}{ Vertical Orientations } \\
\hline Vertical & 32 & 30 & 25 & 25 \\
\hline Horizontal & 17 & 26 & 22 & 20 \\
\hline Oblique & 42 & 35 & 35 & 48 \\
\hline Circular & 9 & 9 & 6 & 8 \\
\hline \multicolumn{5}{|c|}{ Horizontal Orientations } \\
\hline Vertical & 34 & 32 & 34 & 33 \\
\hline Horizontal & 37 & 35 & 22 & 28 \\
\hline Oblique & 27 & 30 & 42 & 37 \\
\hline Circular & 1 & 2 & 2 & 4 \\
\hline \multicolumn{5}{|c|}{ Oblique Orientations } \\
\hline Vertical & 25 & 22 & 24 & 32 \\
\hline Horizontal & 28 & 25 & 26 & 35 \\
\hline Oblique & 45 & 50 & 49 & 30 \\
\hline Circular & 1 & 2 & 2 & 2 \\
\hline
\end{tabular}

category by an individual were therefore converted to percentages of the total score in each condition for that individual. The results for the 12 subjects ( 4 in each condition) are shown in Table 3.

The results show that the percentage of circular movements was by far lower than that of any of the straight movements in all conditions. Oblique movements predominated. The patterns of movements were similar in all conditions for open and closed shapes. The total number of movements scored for the 12 subjects was 7,928. Subjects consistently produced more movements for open $(4,366)$ than for closed shapes $(3,562)$.

\section{Discussion}

Symmetry had no significant effect on either errors or latency of responses, although exploration time was unlimited and accuracy was high (96\%). Lack of facilitation from bilateral symmetry cannot, therefore, be attributed to poor tactual discrimination. The lack of any effect of symmetry was expected, on the grounds that one-handed exploration of small shapes in blind conditions fails to provide adequate spatial-reference cues early in tactual processing.

The finding that judgments for closed shapes were less accurate but faster, whereas those for open shapes showed the reverse, suggests a speed-accuracy tradeoff. Closed shapes also produced fewer movements. But $S D$ s for RTs for closed versus open shapes (2.432 vs. 2.745$)$ were not consistent with the larger $S D$ s typical of self-terminating responses. More important, self-termination of scanning cannot explain the absence of symmetry effects, since open shapes showed no symmetry effect either. That symmetry effects were absent because of ceiling-level accuracy was also considered unlikely, because symmetry should have reduced latencies if the comparative lack of spatial reference were not involved. To check on that possibility, ceiling-level accuracy was controlled in the next experiment.

These findings contrast with the highly significant effects of bilateral symmetry found for vision (Experiment 1), although the task did not explicitly require the detection of symmetry in either experiment. With explicit instructions, the symmetry/asymmetry dimension can be detected by touch (see, e.g., Ballesteros, 1993; Locher \& Simmons, 1978), though asymmetric judgments tend to be better with 2-D raised-line stimuli. The lack of symmetry/asymmetry effects for touch here replicates the results of an earlier study with similar stimulus conditions and an indirect perceptual task (Millar, 1978).

Our task was deliberately designed to avoid explicit instructions, in order to test whether symmetry effects can be regarded as part of early processing in tactual shape perception. If bilateral symmetry were an early organizing property in touch, regardless of available spatial-reference information, an effect on latencies would have been expected. However, we restricted exploration time in the next experiment in order to control for the possibility that 
the lack of significant symmetry effect in this experiment was due to the near ceiling-level accuracy of responses.

The aim of the next experiment was to compare the effects of bilateral symmetry in tactual conditions that provide spatial reference with the effects in the one-finger condition used in the present experiment. The same indirect judgment task was used. Presentation times were restricted in order to avoid ceiling and floor effects and to obtain accuracy levels comparable to those in the visual experiment.

A pilot study was run to achieve accuracy levels that avoided floor and ceiling effects and that were comparable to the level of accuracy in the visual experiment, in which viewing time had also been restricted (Experiment 1).

\section{EXPERIMENT 3}

The aim of Experiment 3 was to provide subjects with the possibility of using the body midline as a spatialreference frame for parallel, conjoint (simultaneous) exploration of the stimuli by the forefingers of the two hands. It was argued that aligning the starting position of the two forefingers to the midaxis of the body and stimulus cards, perpendicularly above the stimuli, could act more sensitively to provide spatial-reference information for scanning bilateral stimuli. The hypothesis predicts that the two-forefinger condition, but not the one-finger condition, would show a significant advantage for symmetric over asymmetric vertical stimuli. Fewer or no symmetry advantages were expected for horizontal and oblique stimuli, in analogy with the visual findings.

The main manipulation was to compare the twoforefinger condition, in which the position of the two adjacent forefingers was initially aligned to the midpoint of the body, with the one-finger condition that was the same as that in Experiment 2. The point of the two-finger condition was to align the forefingers of the subject so that the body midaxis could provide a spatial-reference anchor for parallel exploratory movements by the two forefingers.

The prediction was that aligning the position of the two forefingers to the body midaxis and midline of the stimulus card would produce a significant advantage for vertically oriented symmetric patterns, but that that advantage would not be shown in one-finger conditions. Bilaterally symmetric horizontal and oblique stimuli would be expected to have less or no advantage over asymmetric stimuli from two-finger scanning in these reference conditions.

The experiment required the comparison of twoforefinger with one-forefinger scanning. The finger conditions thus constituted an added factor. As in the first two experiments, the task was to judge whether the stimulus contained a closed shape.

Because the accuracy of detecting open and closed shapes in Experiment 2 was near ceiling level, a pilot study was run with 5 subjects in order to find a level of accuracy that would avoid floor- and ceiling-level effects and that would be comparable with earlier studies of symmetry and with the level of accuracy found in the visual experiment here. The pilot study showed that an accuracy level of approximately $70 \%$ to $80 \%$ was produced by an exposure time of $1.5 \mathrm{sec}$. That exposure time was consequently used in this experiment.

\section{Method}

Subjects. Forty-eight new right-handed unpaid volunteers receiving course credit at UNED took part. Sixteen subjects were allocated randomly to each of the three orientation conditions. All other variables were within-subjects factors, presented in acrosssubjects counterbalanced order.

Stimuli, Equipment, Procedures, and Design. The task, stimulus sets, apparatus, and main instructions were precisely the same as those in Experiment 2. The stimulus exposure time was limited to $1.5 \mathrm{sec}$. The first tone signalled the start of the trial. The timer started automatically when the subject touched the shape. This was followed by a second tone after $1.5 \mathrm{sec}$. The subjects were asked to lift their finger(s) on hearing the second tone and to respond orally. Lifting the fingers from the shape automatically stopped the timer.

The one-forefinger conditions were precisely the same as those in Experiment 2. The subjects were right-handed and used the preferred hand (see Experiment 2 ). The difference in procedures was in the two-forefinger condition.

In the two-forefinger condition, the subjects were asked to use both forefingers to scan the stimuli. Before every trial, the experimenter carefully positioned the subject's forefingers adjacently in parallel, at a height of approximately $20 \mathrm{~cm}$, directly above the stimulus shape. Great care was taken to ensure that the position of the adjacent forefingers was aligned to the subject's body midaxis. The positioning procedure was carried out immediately prior to the starting signal in each trial. Subjects were told that the shape was directly below the positioned forefingers. They were asked to move the two forefingers perpendicularly down to touch the stimulus pattern immediately on hearing the starting signal and to start scanning. At the start of scanning, therefore, the position of the two forefingers was in direct (vertical in the tabletop plane) line relative to the body midpoint and, therefore, constituted a spatial reference by which stimulus features could be located. In principle, the subjects could scan the stimulus pattern by moving the two forefingers simultaneously in parallel toward the body in the vertical (tabletop plane) direction.

A symmetry/asymmetry $\times$ orientation $(\mathrm{V} / \mathrm{H} / \mathrm{O}) \times$ open/closed stimuli $\times$ one-finger/two-fingers $\times$ smooth-lines/dot-lines stimuli factorial design was used with repeated measures for all except the orientation factor. Orientation was used as a between-subjects variable in this experiment, because the one-finger/two-finger comparison doubled the already large (for touch) number of trials for each subject. There were 4,608 trials in all. Sixteen subjects were allocated randomly to each of the three orientation conditions. Each of the 16 subjects received 96 trials in all, 48 with one-finger exploration and 48 with two-finger exploration in across-subjects counterbalanced order. All other conditions were completely randomized, as before.

\section{Results}

Overall errors. The percentages of errors in all conditions are shown in Table 4. Overall percentages of error ( $26 \%$ overall; $23 \%$ for open, $28 \%$ for closed stimuli) were quite sensitive for our purposes, because they avoided both floor- and ceiling-level effects. This was important, because the unlimited time for tactual exploration in Experiment 2 produced near ceiling-level accuracy for both open and closed shapes. In this experiment, exploration 
Table 4

Mean Percentage of Errors for Open (Op) and Closed (CI) Types of Stimuli in Vertical, Horizontal, and Oblique Orientations, and a Textured or Untextured Reference Line in Exploration by One Finger (1F) or Two Fingers (2F)

\begin{tabular}{|c|c|c|c|c|c|c|c|c|c|c|}
\hline \multirow[b]{3}{*}{ Orientation } & & \multicolumn{4}{|c|}{ Symmetric } & \multicolumn{4}{|c|}{ Asymmetric } & \multirow[b]{3}{*}{$M$} \\
\hline & & \multicolumn{2}{|c|}{ Textured } & \multicolumn{2}{|c|}{ Untextured } & \multicolumn{2}{|c|}{ Textured } & \multicolumn{2}{|c|}{ Untextured } & \\
\hline & & $1 F$ & $2 \mathrm{~F}$ & $1 \mathrm{~F}$ & $2 \mathrm{~F}$ & $1 F$ & $2 \mathrm{~F}$ & $1 \mathrm{~F}$ & $2 \mathrm{~F}$ & \\
\hline \multicolumn{11}{|l|}{ Vertical } \\
\hline & Op & 20.82 & 16.16 & 23.82 & 17.66 & 21.82 & 29.15 & 28.15 & 33.32 & 23.82 \\
\hline & $\mathrm{Cl}$ & 25.99 & 30.15 & 26.99 & 31.15 & 22.82 & 22.82 & 25.99 & 26.99 & 26.66 \\
\hline \multicolumn{11}{|l|}{ Horizontal } \\
\hline & Op & 9.33 & 12.49 & 3.16 & 9.13 & 11.50 & 16.66 & 12.49 & 20.82 & 11.99 \\
\hline & $\mathrm{Cl}$ & 29.15 & 28.15 & 31.32 & 30.15 & 30.15 & 25.98 & 24.99 & 26.98 & 28.32 \\
\hline \multicolumn{11}{|l|}{ Oblique } \\
\hline & Op & 33.32 & 37.85 & 40.32 & 37.98 & 41.65 & 31.99 & 34.49 & 31.99 & 36.15 \\
\hline & $\mathrm{Cl}$ & 27.32 & 33.32 & 24.99 & 26.16 & 17.83 & 28.49 & 29.66 & 43.82 & 28.99 \\
\hline$M$ & & 23.36 & 26.49 & 21.17 & 25.49 & 24.32 & 25.83 & 27.82 & 30.65 & 25.66 \\
\hline
\end{tabular}

time was restricted for that reason. Latencies were consequently not a dependent measure here.

The overall orientation $(\mathrm{V} / \mathrm{H} / \mathrm{O}) \times$ symmetry/asymmetry $X$ open/closed stimuli $X$ one/two fingers $\times$ smoothline/dot-line ANOVA produced a significant main effect only of orientation $\left[F(2,45)=10.398, M S_{e}=3.329, p<\right.$ .0002 ]. Judgments of obliques were less accurate overall ( $33 \%$ errors) than were those of verticals and horizontals, which did not differ from each other $(25 \%$ and $26 \%$, respectively). But orientation also interacted with open/ closed stimuli $\left[F(2,45)=5.811, M S_{\mathrm{e}}=5.355, p<.006\right]$. The interaction between orientation $\times$ open/closed $\times$ fingers was also significant $\left[F(2,45)=3.842, M S_{\mathrm{e}}=1.057\right.$, $p<.03]$. Orientation also interacted with symmetry asymmetry $\times$ open/closed stimuli $\times$ fingers $[F(2,45)=$ $\left.5.65, M S_{\mathrm{e}}=0.596, p<.007\right]$. Finally, there was also an interaction of orientation with symmetry/asymmetry $x$ open/closed stimuli $\times$ smooth-line/dot-line stimuli.

To gain a clear picture of the actual relations between variables that were indicated by the interactions between shape orientation and all other factors, separate ANOVAS were run for each of the three orientations.

Vertical orientation. The ANOVA for the vertical orientation showed a significant interaction of symmetry with open/closed stimuli $\left[F(1,15)=13.80, M S_{\mathrm{e}}=0.599\right.$, $p<.003$ ]. Symmetry effects were significant only for open stimuli. The interaction of symmetry $\times$ open/closed stimuli with one-finger/two-fingers was significant $\left[F(1,15)=5.87, M S_{\mathrm{e}}=0.599, p<.03\right]$. As predicted, symmetric shapes produced significantly fewer errors than did asymmetric shapes for the two-finger conditions ( $16.91 \%$ vs. $31.24 \%)$ but not for one-finger conditions $(22.32 \%$ vs. $24.99 \%)$. But the finding obtained only for open stimuli.

Horizontal orientation. The ANOVA for the horizontal orientation showed no effect of symmetry or of fingers, and none had been expected for that orientation. The only significant main effect was for open/closed stimuli $[F(1,15)$ $\left.=16.49, M S_{\mathrm{e}}=3.7018, p<.001\right]$. There were significantly fewer errors for open (12\%) than for closed (28\%) stim- uli. No other effect or interaction was significant for this orientation.

Oblique orientation. The ANOVA for the oblique orientation showed an interaction between one-finger/twofingers and open/closed stimuli $\left[F(1,13)=9.85, M S_{\mathrm{e}}=\right.$ $0.5553, p<.008]$ and a further interaction between fingers $X$ open/closed stimuli and symmetry/asymmetry $\left[F(1,13)=5.69, M S_{\mathrm{e}}=0.5718, p<.03\right]$. For closed stimuli, one-finger scanning was better than two-finger scanning for both symmetric ( $26.16 \%$ vs. $29.74 \%)$ and asymmetric $(23.75 \%$ vs. $36.16 \%)$ shapes. For open stimuli, errors in one-finger versus two-finger scanning for symmetric ( $36.82 \%$ vs. $37.92 \%)$ and asymmetric $(38.07 \%$ vs. $34.07 \%$ ) shapes were inconsistent.

There was also a higher order interaction between smoothline/dot-line stimuli, symmetry/asymmetry and open/closed stimuli $\left[F(1,15)=7.44, M S_{\mathrm{e}}=1.1102, p<.02\right]$. The difference between smooth-line and dot-line stimuli was not significant for open stimuli. For closed stimuli, dot lines produced more errors in symmetric than in asymmetric patterns $(p<.05)$ and fewer errors than did smooth lines in asymmetric patterns $(p<.05)$.

\section{Discussion}

For open stimuli in the vertical orientation, though not for closed stimuli, the results were consistent with the hypothesis that vertical-axis bilateral symmetry would show an advantage in touch when initial spatial-reference cues were provided. Reference-frame cues were provided here by positioning the two forefingers in line with the body midaxis and centering the adjacent forefingers directly above the stimulus card. The perpendicular downward movement to touch the stimulus positioned the two forefingers on the stimulus in straight vertical (tabletop plane) alignment with the midpoint of the subject's body in that plane (see Method). Symmetric vertical shapes were processed more accurately only in the two-forefingeralignment condition, while the one-forefinger condition showed no symmetry effect, which replicates the findings of Experiment 2. The predicted results for vertical shapes 
were thus found, although they were confined to open stimuli.

The complex higher order interactions for oblique directions were difficult to interpret. However, no consistent symmetry advantage had been predicted for oblique or horizontal stimuli. Dot-line effects were found only for closed oblique symmetric stimuli, for which they produced more errors. The effect does not, in any case, suggest a symmetry advantage. The intention in including stimuli with dot lines in the relevant directions had been to provide additional, shape-based, orientation information in order to produce something like the redundancy of reference information that is available in vision. In fact, the dotline markings interfered with, rather than helped, processing. The factor was therefore dropped from the next study.

An important consideration in designing the stimuli that were oriented in the three $(\mathrm{V} / \mathrm{H} / \mathrm{O})$ directions had been to make the stimulus orientations as clear as possible within the patterns. The stimuli had thus been designed specifically to ensure that as many of the component lines as possible in each stimulus ran in the relevant $(\mathrm{V} / \mathrm{H} / \mathrm{O})$ orientation (see Method, Experiment 1$)$. The next experiment was designed to control for the effects of shape-centered directional lines in the stimuli by using a single set of stimuli and by testing for orientation effects by rotating that set only.

The next experiment had two aims. It was designed to check out whether the advantage of bilateral symmetry for vertical stimuli in the two-finger condition could be replicated, at least for open stimuli. As far as we know, no one has previously found any advantage for bilateral symmetry with unfamiliar tactual stimuli in tasks that did not demand explicit symmetry judgments. To probe the effects of stimulus orientation with stimuli that were not designed to enhance differences in stimulus orientation, only the vertical set of stimuli was rotated to the three orientations, and orientation was to be a within-subjects factor.

\section{EXPERIMENT 4}

The hypothesis was, as before, that providing reference information by aligning the stimulus card and the starting position of the two forefingers to the midpoint of the subject's body midline produces an advantage for bilateral symmetry in touch. The aim was to test whether the symmetry advantage with two-forefinger exploration would be replicated, at least for open stimuli. An advantage for open vertical stimuli would be predicted as before, if component orientational lines in the specially designed stimuli (stimulus sets, Experiment 1) did not add more to orientation effects than did stimulus orientations that are produced solely by rotating the same set to the relevant three orientations.

\section{Method}

The design was simplified by using only two-handed scanning and only smooth-line stimuli. This allowed us to use a within-subjects design on all factors.

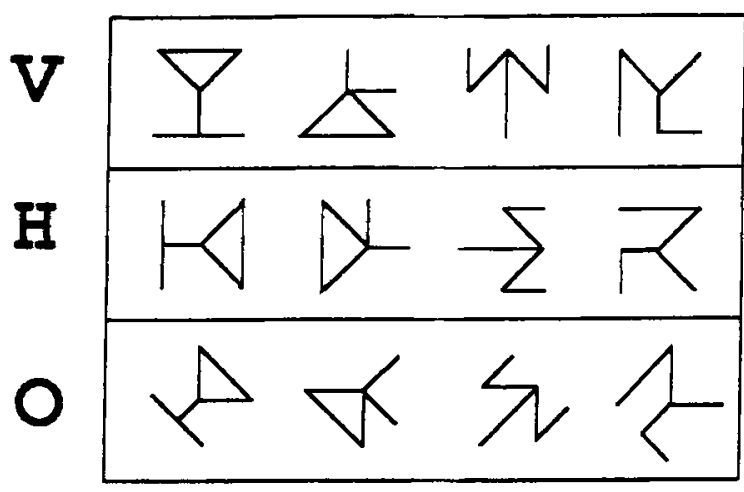

Figure 2. Examples of the set of stimuli used in Experiment 4 in $V$ (vertical), $H$ (horizontal), and $O$ (oblique) orientations. The sequence of stimuli in each orientation is shown as follows: (1) closed symmetric, (2) closed asymmetric, (3) open symmetric, and (4) open asymmetric.

Subjects. Nine new young adult unpaid volunteers (UNED undergraduates, receiving course credit) took part in the experiment. All were right-handed. The number of subjects was sufficient, because each subject was tested in all factorial conditions. Subjects were tested blindfolded and did not see the stimuli or apparatus at any time.

Apparatus, Stimuli, and Procedure. The equipment and procedures were the same as those in Experiment 3 in the two-forefinger condition.

A total of 72 stimuli were prepared, using smooth-line stimuli only, by the same procedures as before. There were 24 stimuli in each of the three orientations. The vertical set was the same as that used in all the previous experiments. For the horizontal set, the same stimuli were rotated by $90^{\circ}$ on the presentation card. For the oblique set, the stimuli were rotated by $45^{\circ}$ on the presentation card. The production of the stimuli on the thermoform presentation cards (see Method, Experiment 1) and all dimensions were precisely the same as those described in Experiments 2 and 3, except that only the smooth-line stimuli were used. Examples of the stimuli are shown in Figure 2.

The three orientation sets were presented in blocked, acrosssubjects counterbalanced order. Within sets, the stimuli were randomized (by computer programming) for every subject, as before. Exposure time $(1.5 \mathrm{sec})$ and signals were the same as those for Experiment 3 .

A symmetric/asymmetric $\times$ orientation $(\mathrm{V} / \mathrm{H} / \mathrm{O}) \times$ open/closed stimuli design was used with repeated measures on all factors. The task was to judge whether the stimuli contained a closed shape, as before; there were 6 trials in each experimental condition. Each subject had a total of 72 trials. The subjects were asked to use the forefingers of both hands for exploration. The experimenter aligned the two forefingers of the subjects prior to each trial, as described in Experiment 3.

Table 5

Mean Percentage of Errors for Types of Stimuli (Open/Closed), Symmetric Versus Asymmetric Conditions in Vertical, Horizontal, and Oblique Orientations

\begin{tabular}{lcccccc}
\hline & \multicolumn{2}{c}{ Open } & & \multicolumn{2}{c}{ Closed } & \\
\cline { 2 - 3 } \cline { 5 - 6 } Orientation & Symmetric & Asymmetric & & Symmetric & Asymmetric & $M$ \\
\hline Vertical & 14.83 & 23.99 & & 20.32 & 18.49 & 19.49 \\
Horizontal & 16.66 & 22.07 & & 35.15 & 20.32 & 23.65 \\
Oblique & 18.49 & 31.49 & & 33.32 & 29.55 & 28.32 \\
$M$ & 16.66 & 24.99 & & 29.65 & 22.82 & 23.82 \\
\hline
\end{tabular}




\section{Results}

Errors. Mean percentage errors are shown in Table 5. The percentage of errors for both open $(21 \%)$ and closed (26\%) shapes was similar to that in the previous experiment and showed neither floor- nor ceiling-level effects, as had been intended by limiting exploration time.

The symmetry/asymmetry $\times$ open/closed stimuli $X$ orientation (V/H/O) ANOVA showed no significant main effects. The effect of orientation showed some tendency for errors to be lower for vertical than for horizontal orientations and highest for obliques, as shown in Table 4. But the effect was not significant $\left[F(2,16)=1.86, M S_{\mathrm{e}}=\right.$ $2.5093, p=.1855$ ], and, unlike the results in Experiment 3 , orientation did not interact significantly with any other factor. Orientation was manipulated here by rotating the vertical set of stimuli.

Symmetry/asymmetry interacted significantly with open/closed stimuli $\left[F(1,8)=11.81, M S_{\mathrm{e}}=0.53, p<\right.$ $.009]$. As in Experiment 3, for open shapes only, errors were significantly lower for symmetric than for asymmetric open shapes ( $t$ test, $p<.05$ ). For closed shapes, the difference was in the opposite direction, although not significantly so $(p=.10)$.

Analysis of finger movements. In order to get a clearer idea of the subjects' movement strategies, the finger movements for 4 (randomly selected) of the 9 subjects who took part in this experiment were scored in a frameby-frame analysis by an independent observer. The criteria and scoring of movements were the same as those in Experiment 2. Because only two-finger exploration was used in this experiment, the scorer was asked to look out for any data that showed independent rather than conjoint movements by the two forefingers. A conjoint movement in this context means that the subjects used the adjacent forefingers in parallel movements rather than independently of each other. In fact, independent movements by the two forefingers were found only for 1 subject. This subject also was the only one to use one finger as an an-

Table 6

Mean Percentage of Coordinated Vertical, Horizontal, Oblique, and Circular Movements for Open and Closed, Symmetric and Asymmetric Types of Stimuli in Vertical, Horizental, and Oblique Orientations (Experiment 4)

\begin{tabular}{|c|c|c|c|c|}
\hline \multirow[b]{2}{*}{ Movement } & \multicolumn{2}{|c|}{ Open } & \multicolumn{2}{|c|}{ Closed } \\
\hline & Symmetric & Asymmetric & Symmetric & Asymmetric \\
\hline \multicolumn{5}{|c|}{ Vertical Orientations } \\
\hline Vertical & 32 & 24 & 29 & 19 \\
\hline Horizontal & 27 & 35 & 33 & 33 \\
\hline Oblique & 26 & 32 & 30 & 34 \\
\hline Circular & 13 & 7 & 9 & 8 \\
\hline \multicolumn{5}{|c|}{ Horizontal Orientations } \\
\hline Vertical & 45 & 32 & 35 & 22 \\
\hline Horizontal & 20 & 20 & 20 & 27 \\
\hline Oblique & 19 & 34 & 31 & 41 \\
\hline Circular & 14 & 15 & 15 & 10 \\
\hline \multicolumn{5}{|c|}{ Oblique Orientations } \\
\hline Vertical & 20 & 34 & 29 & 29 \\
\hline Horizontal & 22 & 16 & 12 & 20 \\
\hline Oblique & 40 & 37 & 50 & 34 \\
\hline Circular & 6 & 12 & 10 & 11 \\
\hline
\end{tabular}

chor (stationary on a feature) while exploring the remainder with the other finger for approximately half $(40 \%)$ of all movements, which suggests that the subject was using one finger as a spatial anchor. Most of the other disjoint movements $(38 \%)$ by this subject were not clearly classifiable. The remainder of his movements $(22 \%)$ were conjoint movements like those found consistently for the other 3 subjects. Table 6 shows the mean percentage of scores for conjoint vertical, horizontal, oblique, and circular movements for these subjects.

The percentage of circular movements was again very low in all conditions. The highest percentage of oblique movements was found for the obliquely oriented stimuli. The patterns of movements suggest that orientation had some effect for symmetric open stimuli. However, horizontally oriented open symmetric shapes showed a larger proportion of vertical than of horizontal movements, and the symmetry $X$ orientation interaction in the accuracy results was not significant.

The movement patterns for closed symmetric and asymmetric shapes and for asymmetric open shapes were similar. There were no differences in the total number of conjoint movements for open (509) as compared with closed (511) shapes in this study.

\section{Discussion}

The prediction that symmetric shapes would show an advantage when the two forefingers were aligned to the body midline and stimuli at the start of scanning was again supported, but, again, for open stimuli only, as in the previous experiment in the two-forefinger condition. The use of conjoint movements as such could not explain why symmetry facilitated the processing of open but not of closed stimuli, because the two types of shape showed the same movements.

The advantage of symmetric open shapes over asymmetric open shapes was not confined to the vertical orientation of stimuli. Thus, stimulus orientation was not significant when orientation effects were tested by rotating the vertical stimulus set to the other orientations, instead of by using stimuli that were specifically designed also to contain as many component lines in the relevant directions as possible. Thus, when orientation depended only on rotating the vertical set to horizontal and oblique conditions, symmetric open shapes showed an advantage over asymmetric open shapes in all three orientation conditions.

\section{SUMMARY AND GENERAL DISCUSSION}

The experiments tested the hypothesis that the advantage of bilateral symmetry in the perception of 2-D shapes can largely be explained by the amount and type of spatial-reference information that is initially available in processing. It was tested by looking for symmetry effects in an indirect perceptual task. The explicit task was to detect a closed shape.

The first experiment supported the suggestion in the literature that bilateral symmetry is an encoding property for 2-D shapes in vision. Symmetry facilitated processing, al- 
though the task did not demand symmetry detection explicitly. Judgments for closed shapes were more accurate than those for open shapes. Vertical-axis bilateral symmetry was superior, as in most previous reports on visual mirror-image symmetry.

The same task and stimuli showed no effects of symmetry for touch in the one-finger condition (Experiment 2), although exploration time was unlimited. Closed shapes produced more errors. But accuracy was near ceiling level for closed as well as for open stimuli. The lack of effects of symmetry and also of orientation cannot, therefore, be attributed to poor tactual recognition. On the other hand, the lack of symmetry effect had been predicted for an indirect task that did not explicitly demand a search for symmetric properties in one-finger scanning conditions. The assumption was that one-finger exploration of novel small 2-D tactual patterns affords few reference cues for organizing inputs spatially as global shapes. The findings are consistent with that assumption. Symmetry does not seem to be an encoding property for touch in these conditions.

By contrast, a significant symmetry advantage was found for vertical open stimuli in the two-forefinger condition that had been designed to provide spatial-reference cues, whereas the one-finger condition with which it was compared again showed no symmetry effects (Experiment 3). In the two-forefinger condition, an initial bodycentered reference frame was provided by aligning the two forefingers to the subject's body midline, centered directly above the stimulus pattern. A perpendicular downward movement ensured that the two forefingers touched the stimulus at a location that was in direct line with the midaxis of the body at the start of scanning. The task condition thus provided reference frame information, rather than acting to elicit or constrain movements. The one-finger condition was the same as that in Experiment 2. The finding for vertical open stimuli in the two-finger conditions is thus consistent with the hypothesis. But the finding was confined to open shapes.

The final experiment again showed a significant advantage for symmetric open stimuli over asymmetric open stimuli in the two-forefinger conditions with the same indirect task. As before, the symmetry advantage was confined to open shapes. Further, the advantage for symmetric open stimuli versus asymmetric open shapes was found for all stimulus orientations to which the vertical stimulus set was rotated. This was in contrast to Experiment 3 , which tested for orientation effects with stimuli that had been specially designed with as many lines as possible in the relevant orientation and which showed the symmetry advantage only for open vertical stimuli in the relevant (two-finger) condition.

Possible reasons for the finding that only open stimuli showed the expected symmetry advantage in two-finger conditions are considered first. The small size of the component shape in closed stimuli can be discounted. The task was to detect that shape, and the detection accuracy was comparable in touch (Experiments 3 and 4 ) and vision (Ex- periment 1) when exposure times were restricted to produce above-floor- and below-ceiling-level accuracy. To our knowledge, no other study has tested symmetry effects with the same stimuli in touch and vision at comparable levels of accuracy. Lower accuracy in tactual than in visual shape perception is often held to explain differences in the effects of other variables. That is not appropriate here. Moreover, Loomis (1993), who reduced visual acuity and viewing apertures to match efficiency levels in touch in a different task, also found that equal acuity levels do not necessarily produce precisely the same effects in touch and in vision. In the present study, the component closed shape was actually larger in area than was the total braille matrix.

It is also unlikely that the failure to detect symmetry in closed shapes occurred because the two forefingers interfered with each other in scanning closed shapes. Scanning with two adjacent forefingers is not, as such, necessarily better than using one finger for exploring small shapes. Moreover, one of our findings, that one-finger scanning was better for closed oblique stimuli (Experiment 3 ), suggested interference of this kind. However, that could not explain the lack of symmetry advantage for one-finger scanning of closed stimuli in either that or any other condition (Experiments 2 and 3), any more than did twofinger scanning of closed shapes (Experiments 3 and 4). The lack of symmetry effects for closed as compared with open shapes also cannot be explained in terms of self-terminating versus exhaustive search. Unlimited exploration time with one finger produced a speed-accuracy tradeoff, but no symmetry effects for either type of shape (Experiment 2). When exploration time was limited (Experiments 3 and 4), the subjects did not terminate the search early for either shape, and the number of scanning movements did not differ (Experiment 4). Yet only open shapes in the two-forefinger condition produced symmetry effects.

The types of scanning movement that the subjects used may have had contributory effects. The relative paucity of circular movements and the predominance of straight vertical and oblique scanning movements for closed as well as for open shapes suggests that scanning movements were not primarily elicited here by stimulus features. Moreover, the straight movements clearly did not prevent the detection of the closed shape. But though closed shapes had the same actual number of lines, fewer of these lines were open-ended than those in open shapes. The combination of straight-line scanning and fewer openended lines in closed shapes may help to explain the failure to detect an incidental shape property like symmetry in closed shapes. Nevertheless, the advantage of symmetry for open shapes cannot be explained without taking account of the reference information provided by the twoforefinger condition, because it was only in that condition that symmetry effects for open shapes were found.

The reason for the predominant use of straight up-down and oblique strokes is more likely to be found in subjectdetermined strategies. Such scanning is often shown in shape recognition by adults who have learned braille relatively recently. They can be quite accurate, though expe- 
rienced braillists use far more highly organized and differentiated scanning strategies (Millar, 1997). The importance of practice and experience for task-adapted scanning strategies is not in doubt. Blind adults are better than blindfolded sighted people at using the two hands in parallel to recognize shape and orientation (see, e.g., Foulke \& Warm, 1967; Warm \& Foulke, 1968). Experienced subjects are likely to use the two forefingers in parallel to scan the sides of a shape (see, e.g., Lappin \& Foulke, 1973) for explicit judgments of symmetry.

However, the question here was, specifically, how to facilitate the incidental detection of shape symmetry by touch without extended prior experience or explicit search. The interesting point is that shape symmetry was actually detected as an incidental property in the relevant condition, though not in all conditions.

The findings on orientation effects require explanation in terms of the composition of the stimuli. The assumption that shape-centered orientation lines within the stimuli would enhance orientation effects seems to have been borne out. Experiment 4 was designed to control for that and to test orientation effects when only the vertical stimuli were rotated. This reduced the orientation effect and showed symmetry advantages for open stimuli in all orientations.

The symmetry and orientation effects in the two experiments (Experiments 3 and 4) thus suggest that the facilitating effects of shape symmetry in touch depend on the redundancy of information from all sources. The vertical lines in the specially designed open stimuli were compatible with body-centered frame cues; the horizontal and oblique lines were discrepant. Rotating the vertical set instead reduced discrepant orientational cues. The interpretation makes sense, but it clearly needs a good deal of work. It raises further questions about the relation between specific stimulus patterns, haptic cues, and spatialframe cues and about the conditions in which they operate redundantly or interfere with each other.

It should be noted, however, that the composition of the vertical stimuli alone cannot explain why the significant advantage for symmetric over asymmetric open stimuli was found only in the two-forefinger conditions (Experiments 3 and 4), but not in the one-finger conditions (Experiments 2 and 3 ). If symmetry advantages had arisen only from the composition of the stimuli, they should have produced the same effects in the one-finger condition.

The point is that visual conditions (Experiment 1) provide spatial-reference information from a variety of external sources, including the viewing aperture and the fixation point, as well as from the fact that the relation between features can be seen "at a glance." Such reference information influences spatial organization and symmetry detection in vision "early in encoding." The latter phrase is used here in the operational sense that symmetrically organized shapes facilitated processing even though the task did not draw attention to symmetry detection at any time. Early encoding does not preclude cogni- tive processes, although these are not necessarily conscious. But the distinction here is the strictly operational one between the incidental concomitants of processing and the explicit task effects.

Operationally, the facilitating effects in touch were incidental to the task. The fact that symmetry facilitated tactual processing, at least of open shapes, when task conditions were designed to provide initial reference cues for spatial organization, suggests that symmetric organization is part of early shape coding processes in touch as well when these conditions obtain.

Taken together, the findings show that shape symmetry can facilitate haptic as well as visual processing of small 2-D patterns, without specific training or explicit demands to search for these properties. That has not been demonstrated in touch before. The effect occurred only in the two-forefinger conditions that had been specifically designed to provide more finely tuned (here body-centered) spatial-reference information than is usually available in touch for unfamiliar small stimuli. The finding is thus consistent with the working assumption that initial spatialreference information is a factor in incidental symmetry advantages in touch. At the same time, the absence of symmetry effects for closed shapes and the orientation effects for stimuli with additional shape-centered orientational lines also suggest interactions with the composition of stimuli that have yet to be unravelled. More complex descriptions, analogous to descriptions of visual symmetry effects (see, e.g., Locher et al., 1993; Wagemans, 1995) will require a great deal more work in order to specify the interactions between the different factors that affect tactual processing.

\section{REFERENCES}

APPELLE, S. (1991). Haptic perception of form: Activity and stimulus attributes. In M. Heller \& W. Schiff (Eds.) The psychology of touch (pp. 169-188). Hillsdale, NJ: Erlbaum.

Ballesteros, S. (1993, May). Haptic perception and types of memory for raised patterns and three-dimensional objects. Paper presented at the conference on object representation in visual and haptic systems, Madrid, Spain.

Ballesteros, S., Manga, D., Navarredonda, A. B., \& Reales, J. M. (1993, November). Perceptual asymmetry in haptic discrimination. Paper presented at the 34th Annual Meeting of the Psychonomic Society, Washington, DC.

Ballesteros, S., Manga, D., \& Reales, J. M. (1997). Haptic discrimination of bilateral symmetry in 2-dimensional and 3-dimensional unfamiliar displays. Perception \& Psychophysics, 59, 37-50.

Ballesteros, S., \& Reales, J. M. (1992, November). The perception of symmetric and asymmetric patterns by touch and vision. Paper presented at the 33rd Annual Meeting of the Psychonomic Society, St. Louis.

BarLow, H. B. (1986). Why we have multiple cortical areas. Vision Research, 26, 81-90.

BaRLow, H. B., \& ReEves, B. C. (1979). The versatility and absolute efficiency of detecting mirror symmetry in random dot displays. Vision Research, 19, 783-793.

Berla, E. P., \& Burterfield, L. H. JR. (1977). Tactual distinctive feature analysis: Training blind students in shape recognition and in locating shapes on a map. Journal of Special Education, 11, 336-346. 
BERTHOZ, A. (1991). Reference frames for the perception and control of movement. In J. Paillard (Ed.), Brain and space (pp. 81-111). Oxford: Oxford University Press.

Bornstein, M. H., Ferdinandson, K., \& Gross, C. G. (1981). Perception of symmetry in infancy. Developmental Psychology, 17, 82-86.

BornsteIN, M. H., \& KRINSKY, S. J. (1985). Perception of symmetry in infancy: The salience of vertical symmetry and the perception of pattern wholes. Journal of Experimental Child Psychology, 39, 1-19.

BruCE, V., \& GREEN, P. (1990). Visual perception, physiology and ecology. Hillsdale, NJ: Erlbaum.

Corballis, M. C., \& Roldan, C. E. (1975). Detection of symmetry as a function of angular orientation. Journal of Experimental Psychology: Human Perception \& Performance, 1, 221-230.

DAVIDSON, P. W. (1972). The role of exploratory activity in haptic perception: Some issues, data and hypotheses. Research Bulletin, American Foundation for the Blind, 24, 21-28.

Davidson, P. W., Aвbott, S., \& Gershenfeld, J. (1974). Influence of exploration time on haptic and visual matching of complex shape. Perception \& Psychophysics, 15, 539-543.

FisHER, C. B., \& BORNSTEIN, M. H. (1982). Identification of symmetry: Effects of stimulus orientation and head position. Perception \& Psychophysics, 32, 443-448.

Fisher, C. B., Ferdinandson, K., \& Bornstein, M. (1981). The role of symmetry in infant form discrimination. Child Development, 52, 457-462.

FoulKE, E., \& WARM, J. S. (1967). Effects of complexity and redundancy on the tactual recognition of metric figures. Perceptual \& Motor Skills, 25, 177-187.

HOWARD, I. P., \& ROGERS, B. J. (1995). Binocular vision and stereopsis. Oxford: Oxford University Press.

HowARD, I. P., \& TEMPLETON, W. B. (1966). Human spatial orientation. New York: Wiley.

JuLESz, B. (1971). Foundations of cyclopean perception. Chicago: University of Chicago Press.

KATZ, D. (1925). Der Aufbau der Tastwelt [The construction of the world of touch]. Leipzig: Barth.

KATZ, D. (1989). The world of touch (L. E. Krueger, Trans.). Hillsdale, NJ: Erlbaum.

KRUEGER, L. E. (1982). Tactual perception in historical perspective: David Katz's world of touch. In W. Schiff \& E. Foulke (Eds.), Tactual perception: A source book (pp. 1-54). Cambridge: Cambridge University Press.

LAPPIN, J. S., \& FoulKE, E. (1973). Expanding the tactual field of view. Perception \& Psychophysics, 14, 237-241.

LEDERMAN, S. J., \& KINCH, D. H. (1979). Texture in tactual maps and graphics for the visually handicapped. Journal of Visual Impairment \& Blindness, 73, 217-227.

Locher, P. [J.], CAVEgn, D., Groner, M., Müller, P. U., D'YDEWALLE, G., \& GRONER, R. (1993). The effects of stimulus symmetry and task requirements on scanning patterns. In G. d'Ydewalle \& J. Van Rensbergen (Eds.), Perception and cognition (pp. 59-69). Amsterdam: Elsevier.

LOCHER, P. J., \& NoDine, C. F. (1973). Influence of stimulus symmetry on visual scanning patterns. Perception \& Psychophysics, 13, 408412.

LOCHER, P. J., \& Simmons, R. W. (1978). Influence of stimulus symmetry and complexity upon haptic scanning strategies during detection, learning, and recognition tasks. Perception \& Psychophysics, 23, $110-$ 116.

LOCHER, P. J., \& Wagemans, J. (1993). Effects of element type and spatial grouping on symmetry detection. Perception, 22, 565-587.

LoOMIS, J. M. (1981). On the tangibility of letters and braille. Perception \& Psychophysics, 29, 37-46.

LoomIS, J. M. (1993). Counterexample to the hypothesis of functional similarity between tactile and visual pattern perception. Perception \& Psychophysics, 54, 179-184.

Loomis, J. M., KLATzKy, R. L., \& Lederman, S. J. (1991). Similarity of tactual and visual picture recognition with limited field of view. Perception, 20, 167-177.
MACH, E. (1897). Analysis of the sensations. Chicago: Open Court.

MARR, D. (1982). Vision: A computational investigation into the human representation and processing of visual information. San Francisco: Freeman.

Millar, S. (1978). Aspects of memory for information from touch and movement. In G. Gordon (Ed.), Active touch: The mechanism of recognition of objects by manipulation: A multi-disciplinary approach (pp. 215-227). Oxford: Pergamon.

MiLLAR, S. (1981a). Crossmodal and intersensory perception and the blind. In R. D. Walk \& H. L. Pick, Jr. (Eds.), Intersensory perception and sensory integration (pp. 281-314). New York: Plenum.

Millar, S. (1981b). Self-referent and movement cues in coding spatial location by blind and sighted children. Perception, 10, 255-264.

MILLAR, S. (1985). Movement cues and body orientation in recall of spatial locations of blind and sighted children. Quarterly Journal of Experimental Psychology, 37A, 257-278.

MiLlar, S. (1991). A reversed lag in the recognition and production of tactual drawings: Theoretical implications for haptic coding. In M. A. Heller \& W. Schiff (Eds.), The psychology of touch (pp. 301-325). Hillsdale, NJ: Erlbaum.

MILLAR, S. (1994). Understanding and representing space: Theory and evidence from studies with blind and sighted children. Oxford: Oxford University Press, Clarendon Press.

Millar, S. (1997). Reading by touch. London: Routledge.

Munsinger, H., \& Forsman, R. (1966). Symmetry, development and tachistoscopic recognition. Journal of Experimental Child Psychology, 3, 168-176.

PallLARD, J. (1991). Motor and representational framing of space. In J. Paillard (Ed.), Brain and space (p. 163-182). Oxford: Oxford University Press.

Palmer, S. E. (1989). Reference frames in the perception of shape and orientation. In B. E. Shepp \& S. Ballesteros (Eds.), Object perception: Structure and process (pp. 121-163). Hillsdale, NJ: Erlbaum.

Palmer, S. E. (1991). Goodness, Gestalt, groups, and Garner: Local symmetry subgroups as a theory of figural goodness. In G. R. Lockhead \& J. R. Pomerantz (Eds.), The perception of structure (pp. 2339). Hillsdale, NJ: Erlbaum.

Palmer, S. E., \& Hemenway, K. (1978). Orientation and symmetry. Journal of Experimental Psychology: Perception \& Performance, 4 , 691-702.

PASHLER, H. ( 1990 ). Coordinate frame for symmetry detection and object recognition. Joumal of Experimental Psychology: Perception \& Performance, 16, 150-163.

Rock, I. (1983). The logic of perception. Cambridge, MA: MIT Press.

ROYER, F. L. (1981). Detection of symmetry. Journal of Experimental Psychology: Human Perception \& Performance, 7, 1186-1210.

SCHIFF, W., KaUfER, L., \& MosaK, S. (1966). Informative tactile stimuli in the perception of direction. Perceptual \& Motor Skills, 23, 13151335.

Simmons, R., \& Locher, P. (1979). Role of extended perceptual experience upon haptic perception of nonrepresentation shapes. Perceptual \& Motor Skills, 48, 987-991.

Van Der Helm, P. A., \& Leeuwenberg, E. L.-J. (1996). Goodness of visual regularities: A nontransformational approach. Psychological Review, 103, 429-456.

WAGEMANS, J. (1995). Detection of visual symmetries. Spatial Vision, 9 , 9-32.

Wagemans, J., Van Gool, L., \& D'Ydewalle, G. (1992). Orientation effects and component processes in symmetry detection. Quarterly Journal of Experimental Psychology, 44A, 475-508.

WALK, R. D. (1965). Tactual and visual learning of forms differing in degrees of symmetry. Psychonomic Science, 2, 93-94.

WARM, J. S., \& FoulKE, E. (1968). Effects of orientation and redundancy on tactual perception of form. Perceptual \& Motor Skills, 27, 83-89.

(Manuscript received March 26, 1996; revision accepted for publication February 19, 1997.) 